\title{
Hadronic Leading Order Contribution to the Muon $g-2$
}

\author{
Daisuke Nomura ${ }^{1,2, a}$ (for the KNT collaboration [1]) \\ ${ }^{1}$ KEK Theory Center, Tsukuba, Ibaraki 305-0801, Japan \\ ${ }^{2}$ Yukawa Institute for Theoretical Physics, Kyoto University, Kyoto 606-8502, Japan
}

\begin{abstract}
We calculate the Standard Model (SM) prediction for the muon anomalous magnetic moment. By using the latest experimental data for $e^{+} e^{-} \rightarrow$ hadrons as input to dispersive integrals, we obtain the values of the leading order (LO) and the next-to-leading-order (NLO) hadronic vacuum polarisation contributions as $a_{\mu}^{\text {had, LO VP }}=(693.27 \pm 2.46) \times 10^{-10}$ and $a_{\mu}^{\text {had, NLO VP }}=(-9.82 \pm 0.04) \times 10^{-10}$, respectively. When combined with other contributions to the SM prediction, we obtain $a_{\mu}(\mathrm{SM})=(11659182.05 \pm 3.56) \times 10^{-10}$, which is deviated from the experimental value by $\Delta a_{\mu} \equiv a_{\mu}(\exp )-a_{\mu}(\mathrm{SM})=(27.05 \pm 7.26) \times 10^{-10}$. This means that there is a $3.7 \sigma$ discrepancy between the experimental value and the SM prediction. We also discuss another closely related quantity, the running QED coupling at the $Z$-pole, $\alpha\left(M_{Z}^{2}\right)$. By using the same $e^{+} e^{-} \rightarrow$ hadrons data as input, our result for the 5-flavour quark contribution to the running QED coupling at the $Z$ pole is $\Delta \alpha_{\text {had }}^{(5)}\left(M_{Z}^{2}\right)=(276.11 \pm 1.11) \times 10^{-4}$, from which we obtain $\alpha^{-1}\left(M_{Z}^{2}\right)=128.946 \pm 0.015$.
\end{abstract}

\section{Introduction}

The anomalous magnetic moment of the muon, $a_{\mu}$, also known as the muon $g-2$, is an extremely important quantity in particle physics since it can be used to probe/constrain new physics beyond the Standard Model (SM). Experimentally, it has been measured to an extremely high precision [2], and the current world average reads [3]

$$
a_{\mu}(\exp )=(11659209.1 \pm 6.3) \times 10^{-10} .
$$

This value should be compared to the SM prediction for $a_{\mu}$. According to Ref. [1], the most recent value of the SM prediction is

$$
a_{\mu}(\mathrm{SM})=(11659182.05 \pm 3.56) \times 10^{-10} .
$$

The difference $\Delta a_{\mu}$ between Eqs. (1) and (2) is

$$
\Delta a_{\mu} \equiv a_{\mu}(\exp )-a_{\mu}(\mathrm{SM})=(27.05 \pm 7.26) \times 10^{-10},
$$

which means a $3.7 \sigma$ discrepancy. This deviation may be due to a contribution from physics beyond the SM, which makes $a_{\mu}$ extremely important. There are two experiments which aim to improve the experimental uncertainty by a factor of $4[4,5]$, which further enhances the importance of this quantity.

\section{Standard Model contribution to $a_{\mu}$}

The SM contribution to the muon $g-2$ can be conveniently separated into three pieces: the QED, electroweak (EW),

\footnotetext{
a e-mail: dnomura@post.kek.jp
}

and hadronic contributions,

$$
a_{\mu}(\mathrm{SM})=a_{\mu}(\mathrm{QED})+a_{\mu}(\mathrm{EW})+a_{\mu}(\mathrm{had}) .
$$

The QED contribution is a perturbatively calculable quantity, and has been calculated up to and including 5loop diagrams [6]:

$$
a_{\mu}(\mathrm{QED})=(11658471.8971 \pm 0.007) \times 10^{-10} .
$$

We should note that the 4-loop contributions are already confirmed by other groups $[7,8]$. In addition, the 5-loop contribution is very small $\left(\sim 0.5 \times 10^{-10}\right)$, and hence the QED contribution does not pose any major problem to the muon $g-2$ in the near future.

The EW contribution is also perturbatively calculable, and the current value reads [9]:

$$
a_{\mu}(\mathrm{EW})=(15.36 \pm 0.10) \times 10^{-10} .
$$

The most problematic contribution is the hadronic contributions. The hadronic contributions can be written as the sum of a few terms:

$$
\begin{aligned}
a_{\mu}(\mathrm{had})= & a_{\mu}^{\mathrm{had}, \mathrm{LO} \mathrm{VP}}+a_{\mu}^{\mathrm{had}, \mathrm{NLO} \mathrm{VP}}+a_{\mu}^{\mathrm{had}, \mathrm{NNLO} \mathrm{VP}} \\
& +a_{\mu}^{\mathrm{had}, \mathrm{LbL}}+a_{\mu}^{\mathrm{had}, \mathrm{NLO} \mathrm{LbL}},
\end{aligned}
$$

where the LO, NLO and NNLO hadronic vacuum polarisation (VP) contributions in the first line can be calculated by using dispersive integrals, whilst to compute the lightby-light (LbL) contributions in the second line we have to rely on hadronic models to some extent.

According to our recent evaluation [1], the values of $a_{\mu}^{\text {had, LO VP }}$ and $a_{\mu}^{\text {had, NLO VP }}$ are

$$
a_{\mu}^{\text {had, LO VP }}=(693.27 \pm 2.46) \times 10^{-10},
$$


Table 1. Breakdown of the SM prediction for the muon $g-2$, together with the experimental value and the deviation between the experimental value and the SM prediction. The numbers are given in units of $10^{-10}$.

\begin{tabular}{l|cl}
\hline QED contributions & $11658471.8971(0.007)$ & Ref. [6] \\
EW contributions & $15.36(0.10)$ & Ref. [9] \\
hadronic contributions & & \\
LO hadronic VP contributions & $693.27(2.46)$ & Ref. [1] \\
NLO hadronic VP contributions & $-9.82(0.04)$ & Ref. [1] \\
NNLO hadronic VP contributions & $1.24(0.01)$ & Ref. [10] \\
hadronic l-by-l contributions & $9.8(2.6)$ & Ref. [11] \\
$\quad$ hadronic l-by-l NLO contributions & $0.3(0.2)$ & Ref. [12] \\
\hline \hline Standard Model prediction, $a_{\mu}(\mathrm{SM})$ & $11659182.05(3.56)$ & Ref. [1] \\
experimental value, $a_{\mu}(\exp )$ & $11659209.1(6.3)$ & Refs. [2, 3] \\
\hline \hline difference, $\Delta a_{\mu}\left(\equiv a_{\mu}(\exp )-a_{\mu}(\mathrm{SM})\right)$ & $27.05(7.26), 3.7 \sigma$ & Ref. [1] \\
\hline
\end{tabular}

and

$$
a_{\mu}^{\text {had, NLO VP }}=(-9.82 \pm 0.04) \times 10^{-10},
$$

respectively. The value of the NNLO hadronic VP contribution quoted in Ref. [10] is

$$
a_{\mu}^{\text {had, NNLO VP }}=(1.24 \pm 0.01) \times 10^{-10} .
$$

For the LbL contributions, we use the value of $a_{\mu}^{\text {had, LbL }}$ in Ref. [11]:

$$
a_{\mu}^{\mathrm{had}, \mathrm{LbL}}=(9.8 \pm 2.6) \times 10^{-10},
$$

and the value of $a_{\mu}^{\text {had, NLO LbL }}$ in Ref. [12]:

$$
a_{\mu}^{\text {had, NLO LbL }}=(0.3 \pm 0.2) \times 10^{-10} .
$$

By adding Eqs. (5), (6), (8), (9), (10), (11) and (12) we obtain the SM prediction in Eq. (2), resulting in the 3.7 $\sigma$ deviation between the experimental value and the SM prediction, Eq. (3), as summarised in Table 1.

By comparing the various contributions to the SM prediction, Eqs. (5), (6), (8), (9), (10), (11) and (12), we note that the uncertainty from the LO hadronic VP contribution is one of the largest. It is therefore extremely important to evaluate this term as accurately as possible. In the next section we discuss the evaluation of the LO hadronic VP contribution in Ref. [1].

\section{Leading Order Hadronic Vacuum Polarisation Contribution to $a_{\mu}$}

In this section we discuss the LO hadronic VP contribution to $a_{\mu}$. We first give an overview of this contribution, and then discuss recent update given in Ref. [1], often comparing the result with the one given in Ref. [13], which is the previous analysis in which two of the KNT collaboration are involved. Below we refer to Ref. [13] as HLMNT.

\subsection{Overview of LO hadronic VP contribution}

The LO hadronic VP contribution can be calculated by using the dispersion integral,

$$
a_{\mu}^{\text {had, LO VP }}=\frac{\alpha^{2}}{3 \pi^{2}} \int_{s_{t h}}^{\infty} \frac{\mathrm{d} s}{s} R(s) K(s),
$$

where $s_{t h}=m_{\pi}^{2}, R(s)$ is the hadronic $R$-ratio,

$$
R(s)=\frac{\sigma_{\mathrm{had}, \gamma}^{0}(s)}{\sigma_{\mathrm{pt}}(s)} \equiv \frac{\sigma_{\mathrm{had}, \gamma}^{0}(s)}{4 \pi \alpha^{2} /(3 s)},
$$

and $K(s)$ is a kernel function. The superscript ' 0 ' means that the cross section should not include VP radiative corrections, and the subscript ' $\gamma$ ' implies that the cross section should be inclusive with respect to final state radiations (FSRs). We have to exclude VP radiative corrections since we must avoid possible double-counting with higher order hadronic diagrams.

To understand the $s$-dependence of the kernel function $K(s)$, it is useful to define another function $\hat{K}(s)$ by

$$
\hat{K}(s) \equiv \frac{3 s}{m_{\mu}^{2}} K(s) .
$$

The function $\hat{K}(s)$ is a monotonically increasing function with $\hat{K}\left(m_{\pi}^{2}\right)=0.40, \hat{K}\left(4 m_{\pi}^{2}\right)=0.63$ and $\hat{K}(s) \rightarrow 1$ for $s \rightarrow$ $\infty$. The factor $1 / s$ in the integrand of Eq. (13), together with the $s$-dependence of $K(s)$ as $K(s)=\hat{K}(s) m_{\mu}^{2} /(3 s)=$ $O(1) \times m_{\mu}^{2} /(3 s)$, makes the low energy hadronic data important.

In Table 2, we show contributions to $a_{\mu}^{\text {had, LO VP }}$ from important channels. As clearly seen from the table, the contribution to the mean value as well as the uncertainty from the $\pi^{+} \pi^{-}$channel completely dominates over the others. This can be seen also from the pie charts, Fig. 1, where the importance of the $\pi^{+} \pi^{-}$channel is represented as the large area of the green region (the region $0.6<\sqrt{s}<0.9$ $\mathrm{GeV}$ ) in the upper panel. It follows that the good input data in this channel is crucial for this analysis. 
Table 2. Contributions from important channels to the dispersion integrals Eqs. (13) and (29). The numbers from the energy region $\sqrt{s} \leq 1.937 \mathrm{GeV}$ include contributions from data as well as near-threshold contributions to $2 \pi, 3 \pi$ and $\pi^{0} \gamma$ channels. The numbers in the table are taken from Ref. [1].

\begin{tabular}{|c|c|c|}
\hline channel & $a_{\mu}^{\text {had, LO VP }} \times 10^{10}$ & $\Delta \alpha_{\text {had }}^{(5)}\left(M_{Z}^{2}\right) \times 10^{4}$ \\
\hline \multicolumn{3}{|c|}{ Contributions from $\sqrt{s} \leq 1.937 \mathrm{GeV}$} \\
\hline$\pi^{+} \pi^{-}$ & $503.84 \pm 1.97$ & $34.27 \pm 0.12$ \\
\hline$\pi^{+} \pi^{-} \pi^{0}$ & $47.80 \pm 0.89$ & $4.77 \pm 0.08$ \\
\hline$K^{+} K^{-}$ & $23.03 \pm 0.22$ & $3.37 \pm 0.03$ \\
\hline$\pi^{+} \pi^{-} 2 \pi^{0}$ & $19.39 \pm 0.78$ & $5.00 \pm 0.20$ \\
\hline $2 \pi^{+} 2 \pi^{-}$ & $14.87 \pm 0.20$ & $4.02 \pm 0.05$ \\
\hline$K_{S}^{0} K_{L}^{0}$ & $13.04 \pm 0.19$ & $1.77 \pm 0.03$ \\
\hline$\pi^{0} \gamma^{L}$ & $4.58 \pm 0.10$ & $0.36 \pm 0.01$ \\
\hline$K K \pi$ & $2.71 \pm 0.12$ & $0.89 \pm 0.04$ \\
\hline$K K \pi \pi$ & $1.93 \pm 0.08$ & $0.75 \pm 0.03$ \\
\hline$\vdots$ & $\vdots$ & : \\
\hline \multicolumn{3}{|c|}{ Contributions from $1.937 \leq \sqrt{s} \leq 11.199 \mathrm{GeV}$} \\
\hline Inclusive channel & $43.67 \pm 0.67$ & $82.82 \pm 1.05$ \\
\hline \multicolumn{3}{|c|}{ Narrow Resonance Contributions } \\
\hline$J / \psi$ & $6.26 \pm 0.19$ & $7.07 \pm 0.22$ \\
\hline$\psi^{\prime}$ & $1.58 \pm 0.04$ & $2.51 \pm 0.06$ \\
\hline$\Upsilon(1 S-4 S)$ & $0.09 \pm 0.00$ & $1.06 \pm 0.02$ \\
\hline \multicolumn{3}{|c|}{ Contributions from $\sqrt{s} \geq 11.199 \mathrm{GeV}$} \\
\hline pQCD & $2.07 \pm 0.00$ & $124.79 \pm 0.10$ \\
\hline Total & $693.27 \pm 2.46$ & $276.11 \pm 1.11$ \\
\hline
\end{tabular}
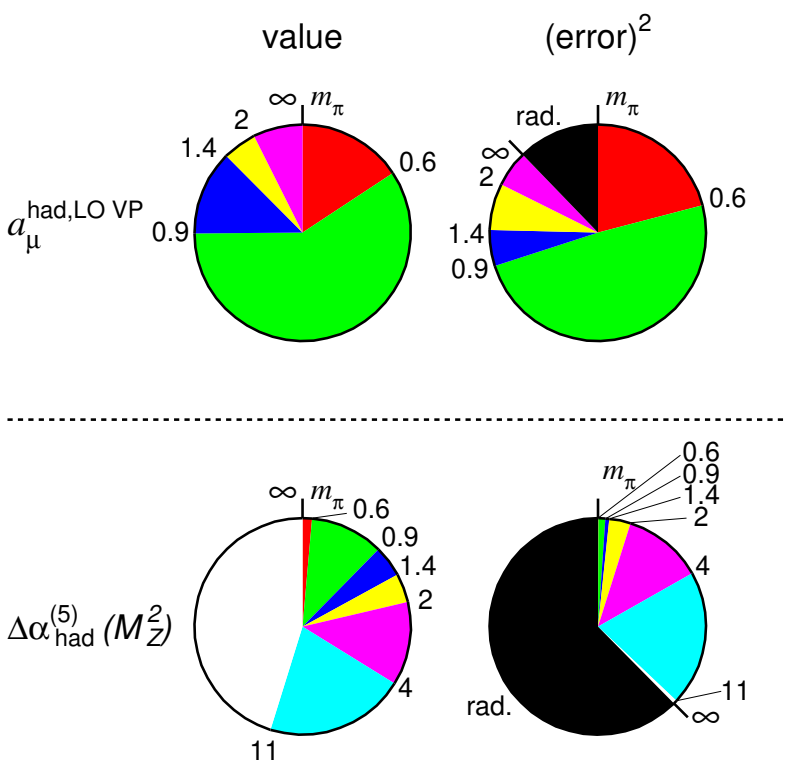

Figure 1. Upper panel: pie charts for the mean value and the error-squared of the LO hadronic VP contribution to the muon

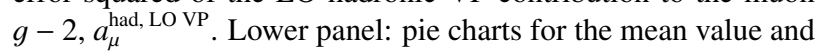
the error-squared of the 5-flavour quark contribution $\Delta \alpha_{\text {had }}^{(5)}\left(M_{Z}^{2}\right)$ to the QED coupling at the $Z$ pole, $\alpha\left(M_{Z}^{2}\right)$.

\subsection{Update in Input Data}

Since the HLMNT paper [13], in the most important $\pi^{+} \pi^{-}$ channel, two major data sets have appeared: one from the KLOE experiment [14], and the other from the BESIII collaboration [15]. To correctly compute the dispersive integral, we have to take into account correlations between data sets. In the case of the KLOE data, there are non-negligible correlations with their previous data sets $[16,17]$. These correlations are correctly summarised in the covariance matrix in Ref. [18], which is used in the KNT analysis [1]. By using these new data sets, together with our improved data-combination algorithm, which we will discuss in the next subsection, we obtain an improvement of more than $30 \%$ in this channel compared to the HLMNT analysis [13], as shown in Table 3.

In the $\pi^{+} \pi^{-} \pi^{0}$ channel, a new data set appeared from the SND collaboration [19], which covers the energy range $1.05 \leq \sqrt{s} \leq 2.00 \mathrm{GeV}$.

In the $K^{+} K^{-}$channel, new precise data has been published from BaBar [20] as well as from CMD-3 [21] and SND [22]. Thanks to these new data, we have about a factor of 2 improvement in the uncertainty compared to the HLMNT analysis.

In the $\pi^{+} \pi^{-} \pi^{0} \pi^{0}$ channel, a new data set appeared from BaBar [23]. In the $\pi^{+} \pi^{-} \pi^{+} \pi^{-}$channel, new data sets have been published from BaBar [24] and CMD-3 [25]. In both channels, these new precise data sets allow us to significantly reduce uncertainties from these channels.

These are just examples of new input data. For a full list of new data, see our paper [1].

\subsection{Improvements in Data Combination Algorithm}

Compared to the HLMNT analysis, we have improved the data combination algorithm.

To evaluate the dispersive integral Eq. (13), it is not a good idea to integrate over raw experimental data. If we would use this method, then the obtained uncertainty of the dispersive integral would be dominated by the contributions of those data points which have large uncertainties. Instead, we should make full use of good accurate data. To do so, we gather near-by data points into a bin (which we call a 'cluster') and integrate over the clusters to evaluate the dispersive integral. In this way, we can reduce influences from inaccurate data points.

In the HLMNT paper, when combining data, the authors assumed a constant cross section across the width of each cluster. In the KNT analysis, we linearly interpolate the cross sections between adjacent clusters, which allows us a more stable determination of the combined cross section. The concrete procedure is the following: We first determine cluster centres $E_{m}$ and the cross section value $R_{m}$ at $E=E_{m}$ by

$$
\begin{aligned}
& R_{m}=\left[\sum_{i=1}^{N^{(m)}} \frac{R_{i}^{(m)}}{\left(\mathrm{d} \tilde{R}_{i}^{(m)}\right)^{2}}\right]\left[\sum_{i=1}^{N^{(m)}} \frac{1}{\left(\mathrm{~d} \tilde{R}_{i}^{(m)}\right)^{2}}\right]^{-1}, \\
& E_{m}=\left[\sum_{i=1}^{N^{(m)}} \frac{E_{i}^{(m)}}{\left(\mathrm{d} \tilde{R}_{i}^{(m)}\right)^{2}}\right]\left[\sum_{i=1}^{N^{(m)}} \frac{1}{\left(\mathrm{~d} \tilde{R}_{i}^{(m)}\right)^{2}}\right]^{-1},
\end{aligned}
$$


Table 3. Difference between the KNT18 analysis [1] and the HLMNT11 analysis [13]. Similarly to Table 2, the numbers from the energy region $\sqrt{s} \leq 2 \mathrm{GeV}$ include contributions from data as well as near-threshold contributions to $2 \pi, 3 \pi$ and $\pi^{0} \gamma$ channels. Note that although the default transition point between the sum of exclusive channels and the inclusive measurement is $1.937 \mathrm{GeV}$ in Ref. [1], in this table we take the transition point at $2 \mathrm{GeV}$ for comparison. This table is extracted from Table 3 of Ref. [1]. For a full table, see Ref. [1].

\begin{tabular}{|l|r|r|r|}
\hline channel & \multicolumn{1}{|c|}{ KNT18 [1] } & HLMNT11 [13] & Diff \\
\hline \multicolumn{4}{|c|}{ Contributions from $\sqrt{s} \leq 2 \mathrm{GeV}$} \\
\hline$\pi^{+} \pi^{-}$ & $503.86 \pm 1.97$ & $505.77 \pm 3.09$ & -2.78 \\
$\pi^{+} \pi^{-} \pi^{0}$ & $47.83 \pm 0.89$ & $47.52 \pm 0.99$ & 0.31 \\
$K^{+} K^{-}$ & $23.05 \pm 0.22$ & $22.15 \pm 0.46$ & 0.90 \\
$\pi^{+} \pi^{-} 2 \pi^{0}$ & $19.80 \pm 0.79$ & $20.37 \pm 1.26$ & -0.57 \\
$2 \pi^{+} 2 \pi^{-}$ & $15.17 \pm 0.21$ & $14.65 \pm 0.47$ & 0.52 \\
$K_{S}^{0} K_{L}^{0}$ & $13.05 \pm 0.19$ & $13.33 \pm 0.16$ & -0.28 \\
$\pi^{0} \gamma$ & $4.58 \pm 0.10$ & $4.66 \pm 0.14$ & -0.08 \\
$K K \pi$ & $2.80 \pm 0.12$ & $2.77 \pm 0.15$ & 0.03 \\
$K K \pi \pi$ & $2.42 \pm 0.09$ & $3.31 \pm 0.58$ & -0.89 \\
\multicolumn{4}{|c|}{ Contributions from $2 \leq \sqrt{s} \leq 11.199 \mathrm{GeV}$} \\
\hline
\end{tabular}

where $E_{i}^{(m)}$ and $R_{i}^{(m)}$ are the energy and the cross section value of the $i$-th data point which gives a contribution to the $m$-th cluster. $\mathrm{d} \tilde{R}_{i}^{(m)}$ is the uncertainty of $R_{i}^{(m)}$ which is defined as

$$
\mathrm{d} \tilde{R}_{i}^{(m)}=\sqrt{\left(\mathrm{d} R_{i ; \mathrm{stat}}^{(m)}\right)^{2}+\left(\mathrm{d} R_{i ; \mathrm{sys}}^{(m)}\right)^{2}},
$$

where $\mathrm{d} R_{i ; \text { stat }}^{(m)}$ and $\mathrm{d} R_{i ; \text { sys }}^{(m)}$ are the statistical and systematic uncertainties of $R_{i}^{(m)}$, respectively. $N^{(m)}$ is the total number of the data points which contribute to the $m$-th cluster. We now assume a linear interpolation between adjacent clusters. In this case, the interpolant cross section value $\mathcal{R}_{m}^{i}$ at $\sqrt{s}=E_{i}^{(m)}$ is given by,

$$
\mathcal{R}_{m}^{i}=\left\{\begin{array}{ll}
R_{m}+\frac{E_{i}^{(m)}-E_{m}}{E_{m+1}-E_{m}}\left(R_{m+1}-R_{m}\right), & \text { if } E_{i}^{(m)}>E_{m} \\
R_{m-1}+\frac{E_{i}^{(m)}-E_{m-1}}{E_{m}-E_{m-1}}\left(R_{m}-R_{m-1}\right), & \text { if } E_{i}^{(m)}<E_{m}
\end{array} .\right.
$$

In addition, we have improved the $\chi^{2}$ function itself. In the HLMNT paper, to construct the $\chi^{2}$ function, the authors take into account the normalisation uncertainties of experimental data by using the 'penalty trick.' Explicitly, the $\chi^{2}$ function used in the HLMNT paper is

$$
\begin{aligned}
& \chi_{\mathrm{HLMNT}}^{2}\left(R_{m}, f_{k}\right)=\sum_{k=1}^{N_{\text {exp }}}\left(\frac{1-f_{k}}{\mathrm{~d} f_{k}}\right)^{2} \\
& +\left\{\sum_{m=1}^{N_{\mathrm{clu}}} \sum_{i=1}^{N_{(k, m)}}\left(\frac{R_{i}^{(k, m)}-f_{k} R_{m}}{\mathrm{~d} R_{i}^{(k, m)}}\right)^{2}\right\}_{\mathrm{w} / \text { out cov. mat. }} \\
& +\sum_{m, n=1}^{N_{\mathrm{clu}}} \sum_{i=1}^{N_{(k, m)}} \sum_{j=1}^{N_{(k, n)}}\left(R_{i}^{(k, m)}-f_{k} R_{m}\right) C^{-1}\left(m_{i}, n_{j}\right)\left(R_{j}^{(k, n)}-f_{k} R_{n}\right),
\end{aligned}
$$

where $f_{k}$ is the overall normalisation factor of the $k$-th experiment. $R_{i}^{(k, m)}$ and $\mathrm{d} R_{i}^{(k, m)}$ are the $R$-values and their errors from $k$-th experiment which contributes to the $m$-th cluster. The authors of the HLMNT paper determined the values of $f_{k}$ and $R_{m}$ by minimising this $\chi^{2}$ function.

Originally, this method is proposed to avoid a potential bias [26-28]. However, in Ref. [29], it is pointed out that this method could also suffer from a potential bias. An alternative way to construct the $\chi^{2}$ function, which is proposed in Ref. [29] and used in the KNT analysis, is to determine the combined cross sections by iteration. We first construct the $\chi^{2}$ function $\chi_{1}^{2}$ for the first iteration as

$$
\chi_{1}^{2}=\sum_{i=1}^{N_{\text {tot }}} \sum_{j=1}^{N_{\text {tot }}}\left(R_{i}^{(m)}-\mathcal{R}_{m}^{i}\right) \mathbf{C}_{1}^{-1}\left(i^{(m)}, j^{(n)}\right)\left(R_{j}^{(n)}-\mathcal{R}_{n}^{j}\right),
$$


where $N_{\text {tot }}$ is the total number of data points. $\mathcal{R}_{m}^{i}$ is the interpolant cross section as a function of $R_{m}$ (see Eq. (19)), and $\mathbf{C}_{1}^{-1}$ is the inverse matrix of the covariance matrix $\mathbf{C}_{1}$ for the first iteration, which is given as the sum of the statistical covariance matrix $C^{\text {stat }}\left(i^{(m)}, j^{(n)}\right)$ and the systematic covariance matrix $C^{\text {sys }}\left(i^{(m)}, j^{(n)}\right)$,

$$
\mathbf{C}_{1}\left(i^{(m)}, j^{(n)}\right)=C^{\mathrm{stat}}\left(i^{(m)}, j^{(n)}\right)+\frac{C^{\mathrm{sys}}\left(i^{(m)}, j^{(n)}\right)}{R_{i}^{(m)} R_{j}^{(n)}} \mathcal{R}_{m}^{i, 0} \mathcal{R}_{n}^{j, 0}
$$

where $\mathcal{R}_{m}^{i, 0}$ is a linearly interpolated cross section value determined by using Eq. (16) as input to Eq. (19). We call the $R_{m}$ values which minimise the function $\chi_{1}^{2}$ as $R_{m}=R_{m}^{1}$, which are used for the input to the next turn of the iterations. From $R_{m}^{1}$, we calculate the interpolant values $\mathcal{R}_{m}^{i}$ by substituting $R_{m}=R_{m}^{1}$ in Eq. (19), and call the output values of $\mathcal{R}_{m}^{i}$ as $\mathcal{R}_{m}^{i, 1}$. We now construct the covariance matrix $\mathbf{C}_{2}$ by

$$
\mathbf{C}_{2}\left(i^{(m)}, j^{(n)}\right)=C^{\mathrm{stat}}\left(i^{(m)}, j^{(n)}\right)+\frac{C^{\mathrm{sys}}\left(i^{(m)}, j^{(n)}\right)}{R_{i}^{(m)} R_{j}^{(n)}} \mathcal{R}_{m}^{i, 1} \mathcal{R}_{n}^{j, 1}
$$

and construct the $\chi^{2}$ function for the second iteration $\chi_{2}^{2}$ as

$$
\chi_{2}^{2}=\sum_{i=1}^{N_{\text {tot }}} \sum_{j=1}^{N_{\text {tot }}}\left(R_{i}^{(m)}-\mathcal{R}_{m}^{i}\right) \mathbf{C}_{2}^{-1}\left(i^{(m)}, j^{(n)}\right)\left(R_{j}^{(n)}-\mathcal{R}_{n}^{j}\right),
$$

where $\mathcal{R}_{m}^{i}$ is the interpolant cross section as a function of $R_{m}$. We find the set of $R_{m}$ which minimises $\chi_{2}^{2}$, which we call $R_{m}^{2}$. Similarly, we construct the $\chi^{2}$ function for the $I$-th iteration as

$$
\chi_{I}^{2}=\sum_{i=1}^{N_{\text {tot }}} \sum_{j=1}^{N_{\text {tot }}}\left(R_{i}^{(m)}-\mathcal{R}_{m}^{i}\right) \mathbf{C}_{I}^{-1}\left(i^{(m)}, j^{(n)}\right)\left(R_{j}^{(n)}-\mathcal{R}_{n}^{j}\right),
$$

where $\mathcal{R}_{m}^{i}$ is the interpolant cross section as a function of $R_{m}$, and

$$
\mathbf{C}_{I}\left(i^{(m)}, j^{(n)}\right)=C^{\mathrm{stat}}\left(i^{(m)}, j^{(n)}\right)+\frac{C^{\mathrm{sys}}\left(i^{(m)}, j^{(n)}\right)}{R_{i}^{(m)} R_{j}^{(n)}} \mathcal{R}_{m}^{i, I-1} \mathcal{R}_{n}^{j, I-1}
$$

We refer to the set of $R_{m}$ which minimises $\chi_{I}^{2}$ as $R_{m}^{I}$. In this way, we repeat this procedure until we reach convergence. In our case, convergence is achieved after only a few steps.

We should note here that since all the $\chi^{2}$ functions Eqs. (21), (24) and (25) are quadratic functions of $R_{m}$, and hence do not introduce a bias [29]. We have also checked that the result of the HLMNT analysis does not change very much even if we adopt the new iterative $\chi^{2}$ minimisation algorithm.

\subsection{Result for LO Hadronic VP Contribution to $a_{\mu}$}

By using the new data input and the new data combination algorithm, we obtain Eq. (8) for $a_{\mu}^{\text {had, LO VP }}$. A similar result has been obtained in Ref. [30], although there are slight differences in some individual channels. Ref. [31] quotes slightly smaller value for $a_{\mu}^{\text {had, LO VP. }}$.

Once the $a_{\mu}^{\text {had, LOVP }}$ is calculated, we can instantly compute $a_{\mu}^{\text {had, NLO VP }}$ since we have only to evaluate similar dispersion relations. Our result for $a_{\mu}^{\text {had, NLO VP }}$ is Eq. (8). When combined with other SM contributions to $a_{\mu}$ such as the QED contribution, we obtain Eq. (2). The difference $\Delta a_{\mu}$ between the experimental determination Eq. (1) and the SM prediction (2) is

$$
\Delta a_{\mu}=a_{\mu}(\exp )-a_{\mu}(\mathrm{SM})=(27.05 \pm 7.26) \times 10^{-10},
$$

which could be due to a contribution from new physics beyond the SM.

\section{Hadronic Contributions to QED Coupling at the $Z$-pole}

Another closely related quantity is the 5-flavour quark contributions $\Delta \alpha_{\text {had }}^{(5)}\left(M_{Z}^{2}\right)$ to the running QED coupling at the $Z$-pole, $\alpha\left(M_{Z}^{2}\right) . \alpha\left(M_{Z}^{2}\right)$ is related to $\Delta \alpha_{\text {had }}^{(5)}\left(M_{Z}^{2}\right)$ by the relation,

$$
\alpha\left(M_{Z}^{2}\right)=\frac{\alpha}{1-\Delta \alpha_{\text {lep }}\left(M_{Z}^{2}\right)-\Delta \alpha_{\text {had }}^{(5)}\left(M_{Z}^{2}\right)-\Delta \alpha_{\text {top }}\left(M_{Z}^{2}\right)},
$$

where $\Delta \alpha_{\text {lep }}\left(M_{Z}^{2}\right)$ and $\Delta \alpha_{\text {top }}\left(M_{Z}^{2}\right)$ are the leptonic and topquark contributions, respectively. While the leptonic and top-quark contributions can be evaluated perturbatively, to evaluate $\Delta \alpha_{\text {had }}^{(5)}\left(M_{Z}^{2}\right)$, we have to rely on the dispersion relation,

$$
\Delta \alpha_{\text {had }}^{(5)}\left(M_{Z}^{2}\right)=-\frac{\alpha M_{Z}^{2}}{3 \pi} \mathrm{P} \int_{s_{t h}}^{\infty} \mathrm{d} s \frac{R(s)}{s\left(s-M_{Z}^{2}\right)} .
$$

Fortunately, this integral can be evaluated by using the same $R$-ratio data as used in the evaluation of $a_{\mu}^{\text {had, LO VP }}$.

By a similar analysis as in the case of $a_{\mu}^{\text {had, LO VP }}$, we obtain

$$
\Delta \alpha_{\text {had }}^{(5)}\left(M_{Z}^{2}\right)=(276.11 \pm 1.11) \times 10^{-4} .
$$

The contributions from major channels are given in Table 2 .

The value of the leptonic contributions is calculated up to and including 4-loop order in Ref. [32], which quotes the value,

$$
\Delta \alpha_{\text {lep }}\left(M_{Z}^{2}\right)=(314.979 \pm 0.002) \times 10^{-4} .
$$

The top-quark contribution is computed in Refs. [33, 34] and the current value is

$$
\Delta \alpha_{\text {top }}\left(M_{Z}^{2}\right)=(-0.7180 \pm 0.0054) \times 10^{-4},
$$

where we have used the values $\alpha_{s}\left(M_{Z}\right)=0.1182(12)$ [3] and $m_{t}=173.1 \pm 0.6 \mathrm{GeV}$ [3] as input parameters. By substituting Eqs. (30), (31) and (32) into Eq. (28), we obtain

$$
\alpha^{-1}\left(M_{Z}^{2}\right)=128.946 \pm 0.015 \text {. }
$$




\section{Conclusions}

We have evaluated the LO and NLO hadronic VP contributions to the muon $g-2$. By using the latest experimental data for $e^{+} e^{-} \rightarrow$ hadrons as input, we obtain the values $a_{\mu}^{\text {had, LO VP }}=(693.27 \pm 2.46) \times 10^{-10}$ and $a_{\mu}^{\text {had, NLO VP }}=(-9.82 \pm 0.04) \times 10^{-10}$, respectively. When combined with other contributions to the SM prediction, we obtain $a_{\mu}(\mathrm{SM})=(11659182.05 \pm 3.56) \times 10^{-10}$, which is deviated from the experimental value by $\Delta a_{\mu}=$ $a_{\mu}(\exp )-a_{\mu}(\mathrm{SM})=(27.05 \pm 7.26) \times 10^{-10}$. This means a $3.7 \sigma$ discrepancy between the experimental value and the SM prediction. This could be due to a contribution from new physics beyond the SM.

We have discussed another closely related quantity, the running QED coupling at the $Z$-pole, $\alpha\left(M_{Z}^{2}\right)$, as well. By using the same $e^{+} e^{-} \rightarrow$ hadrons data as input, our result for the 5-flavour quark contribution to the running QED coupling at the $Z$ pole is $\Delta \alpha_{\text {had }}^{(5)}\left(M_{Z}^{2}\right)=(276.11 \pm 1.11) \times$ $10^{-4}$, from which we obtain $\alpha^{-1}\left(M_{Z}^{2}\right)=128.946 \pm 0.015$.

\section{Acknowledgements}

D. N. thanks the organisers for inviting him to the inspiring workshop in the beautiful surroundings of Capri Island. The work of D. N. is supported by JSPS KAKENHI grant numbers JP16K05323 and JP17H01133.

\section{References}

[1] A. Keshavarzi, D. Nomura and T. Teubner, arXiv:1802.02995 [hep-ph].

[2] G. W. Bennett et al. [Muon $(g-2)$ Collaboration], Phys. Rev. Lett. 92, 161802 (2004); Phys. Rev. D73, 072003 (2006).

[3] C. Patrignani et al. [Particle Data Group], Chin. Phys. C40, 100001 (2016) and 2017 update.

[4] J. Grange et al. [Muon g-2 Collaboration], arXiv:1501.06858 [physics.ins-det].

[5] T. Mibe [J-PARC g-2 Collaboration], Chin. Phys. C34, 745 (2010).

[6] T. Aoyama, T. Kinoshita and M. Nio, Phys. Rev. D97, 036001 (2018).

[7] S. Laporta, Phys. Lett. B772, 232 (2017); these proceedings.

[8] A. Kurz et al., Nucl. Phys. B879, 1 (2014); Phys. Rev. D92, 073019 (2015); Phys. Rev. D93, 053017 (2016).

[9] C. Gnendiger, D. Stöckinger and H. Stöckinger-Kim, Phys. Rev. D88, 053005 (2013).
[10] A. Kurz, T. Liu, P. Marquard and M. Steinhauser, Phys. Lett. B734, 144 (2014).

[11] A. Nyffeler, Phys. Rev. D94, 053006 (2016).

[12] G. Colangelo, M. Hoferichter, A. Nyffeler, M. Passera and P. Stoffer, Phys. Lett. B735, 90 (2014).

[13] K. Hagiwara, R. Liao, A. D. Martin, D. Nomura and T. Teubner, J. Phys. G 38, 085003 (2011).

[14] D. Babusci et al. [KLOE Collaboration], Phys. Lett. B720, 336 (2013).

[15] M. Ablikim et al. [BESIII Collaboration], Phys. Lett. B753, 629 (2016).

[16] F. Ambrosino et al. [KLOE Collaboration], Phys. Lett. B670, 285 (2009).

[17] F. Ambrosino et al. [KLOE Collaboration], Phys. Lett. B700, 102 (2011).

[18] A. Anastasi et al. [KLOE-2 Collaboration], arXiv:1711.03085 [hep-ex].

[19] V. M. Aul'chenko et al. [SND Collaboration], J. Exp. Theor. Phys. 121, 27 (2015).

[20] J. P. Lees et al. [BaBar Collaboration], Phys. Rev. D88, 032013 (2013).

[21] E. A. Kozyrev et al. [CMD-3 Collaboration], Phys. Lett. B779, 64 (2018).

[22] M. N. Achasov et al. [SND Collaboration], Phys. Rev. D94, 112006 (2016).

[23] J. P. Lees et al. [BaBar Collaboration], Phys. Rev. D96, 092009 (2017).

[24] J. P. Lees et al. [BaBar Collaboration], Phys. Rev. D85, 112009 (2012).

[25] R. R. Akhmetshin et al. [CMD-3 Collaboration], Phys. Lett. B768, 345 (2017).

[26] G. D'Agostini, Nucl. Instrum. Meth. A346, 306 (1994).

[27] T. Takeuchi, Prog. Theor. Phys. Suppl. 123, 247 (1996).

[28] V. Blobel, eConf C 030908, MOET002 (2003).

[29] R. D. Ball et al. [NNPDF Collaboration], JHEP 1005, 075 (2010).

[30] M. Davier, A. Hoecker, B. Malaescu and Z. Zhang, Eur. Phys. J. C77, 827 (2017).

[31] F. Jegerlehner, arXiv:1711.06089 [hep-ph].

[32] C. Sturm, Nucl. Phys. B874, 698 (2013).

[33] K. G. Chetyrkin, J. H. Kuhn and M. Steinhauser, Phys. Lett. B371, 93 (1996); Nucl. Phys. B482, 213 (1996); Nucl. Phys. B505, 40 (1997).

[34] J. H. Kuhn and M. Steinhauser, Phys. Lett. B437, 425 (1998). 